\title{
Defects Detection in GFRP Composites by using Transmission method of Infrared Thermography
}

\author{
by M.Y. Choi*, H. S. Park*, K. A. Kwon*, J. H. Park and W.T. Kim* \\ * Safety measurement center, Korea research Institute of Standards and Science, Daejeon, Korea, \\ moonring@kriss.re.kr \\ ${ }^{* *}$ Department of Mechanical Engineering, Kongju National University,Cheonan, Korea
}

\begin{abstract}
In this study, infrared thermography measurement technique has been used to defect detection of composite materials which is widely used in aerospace and wind turbine blades. To increase the defect detection rate, the related experiment used the lock-in IR-thermography method. Unlike the existing reflection method, transmission method that halogen lamp is located behind the specimen has been used to defect detection. The results show that the possibility of detecting defects gets higher than using the transmission method. Infrared thermography images show that all detection of composite material specimen is detected through overall section From $50 \mathrm{mHz}$ to $400 \mathrm{mHz}$.
\end{abstract}

\section{Introduction}

Currently, GFRP(glass fiber reinforced plastic) and CFRP(carbon fiber reinforced plastic) have been widely used as a representative composite industrywide across all industries. The use of these materials has been gradually extended to various application fields such as aerospace, automobile, railroad, building structure and blades for wind power generation. With the increased use of these composite materials, there has been increasing interest in their production processes or detection methods of various defects occurred during use.

Specific defects generated in composite materials are broadly divided into Delamination, Inclusion, Debonding and Void. Although ultrasonic testing has been mainly used as a technique to detect defects of composite materials thus far, it took a long time to detect them and had difficulty detecting defects occurred in the sheet or void defects. In order to solve these problems, infrared thermography testing method has been used recently. This study examined differences between reflection and transmission methods mainly used in testing methods using light source among existing infrared thermography methods through experimentation.

\section{Materials and Experimental Methods}

The GFRP artificial defect specimen in Fig. 1 was composed of 16-layer and made by inserting circular, oval and star-shaped defects into the polytetrafluoroethylene (Teflon) film at 25 and $50 \%$ depth. Inclusion and Debonding defects were included in the defects generated in the specimen. Based on the longitudinal centerline when viewed from the front, the artificial defects on the left side were located in the deepest part, at $25 \%$ depth, and those on the right side in the middle of the specimen, at $50 \%$ depth. Detection methods of defects in composite materials using infrared thermography are broadly divided into active and passive testing methods. In this study, the experiments were performed by applying the active testing method to calculate the defect size by transmitting specific irritants to objects as a harmonic function and processing the reply signals. In order to effectively detect defects of plate composite materials, this study applied the phase lock techniques capable of processing thermography to existing photo-infrared thermography system.[1]. The infrared thermographic testing device was configured with a light source heating device and infrared thermography camera, and in order to minimize heat exchange, the experimental apparatus was composed in the insulation chamber as shown in Fig. 2. Unlike previous methods, $1 \mathrm{~kW}$ light source lamps were set on either side behind the defect specimen, and Silver 480M made by French Cedip was used an infrared camera. For the detection sensitivity of infrared thermography, flat black paint was applied to the surface of the specimen in order to satisfy the conditions similar to the black body with an emissivity of 1 , and the emissivity to was maintained at 0.95.[2]

Table 1. Composite standard specimen

\begin{tabular}{|l|c|}
\hline Material & glass-epoxy unidirectional prepreg (UGN150, SK chemicals) \\
\hline Defect type & Inclusion(Non-perforated release film, 0.001inch thickness) \\
\hline Lay-up sequence & {$\left[\mathrm{O}_{2} / 90_{2} / 45_{2} /-45_{2}\right]_{\S}$} \\
\hline Thickness & $1.4 \mathrm{~mm}$ \\
\hline
\end{tabular}



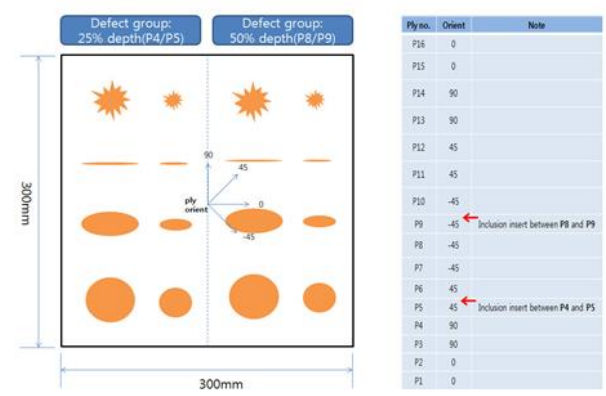

Fig. 1. Inclusion defect specimen of GFRP

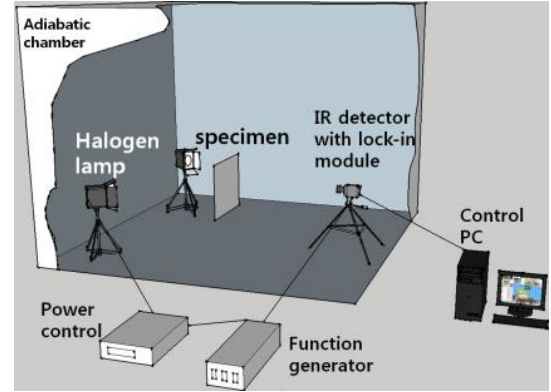

Fig. 2. Infrared thermography experiment system

\section{Experimental Considerations}

\subsection{GFRP Inclusion Defect Specimen}

According to results of the thermography test by the reflection method, the shape of the defect located at $50 \%$ depth was shown in Fig. 3 (a) with light source frequency of $50 \mathrm{mHz}$. In addition, as for the defect located at $25 \%$ depth from the bottom, images to identify approximate location were obtained. It was difficult even to expect the location of the defect at $400 \mathrm{mHz}$. It is considered that this reaction is caused by the principle that the heat penetration deepens in proportion to the heating time of light sources in thermal diffusion.[3] Fig. 3 (b) is a thermographic image to detect defects using the transmission method and shows that the defects at all depths clearly appear even at $400 \mathrm{mHz}$. It is considered difficult to easily detect defects using the reflection method because the defect and parent material were saturated due to energy loss and thermal equilibrium in the process that all heat applied on the surface is reflected and released again after being transferred to the bottom. However, it is considered possible to obtain these results because more energy is transmitted when using the transmission method in heating the specimen using the light source than when using the reflection method and it is possible to detect the transferred energy without reflecting and releasing the heat transfer energy.

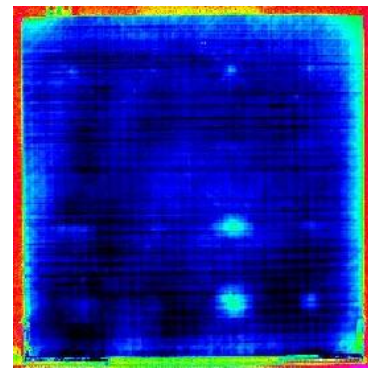

$50 \mathrm{mHz}$

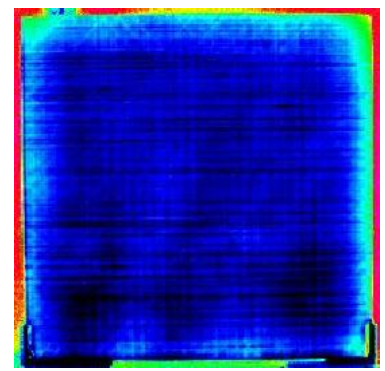

$400 \mathrm{mHz}$

(a) Reflection method

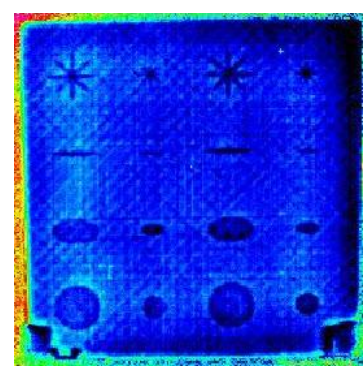

$100 \mathrm{mHz}$

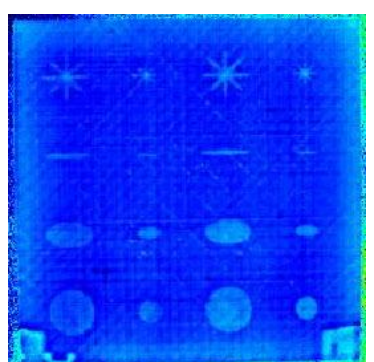

$400 \mathrm{mHz}$

(b) Transmission method

\section{Conclusions}

According to the measurement results by the reflection method, the inclusion and debonding defect specimen with 1.4-mm-thick GFRP composite materials with a 16-layer showed a higher detection rate at $50 \mathrm{mHz}$ with longer lightcuring time than at $400 \mathrm{mHz}$. According to the measurement results by the transmission method to put the light source behind the defect specimen, it was not greatly affected by the light-curing time, and clear defect images were obtained.

\section{REFERENCES}

[1] G. Busse, "Infrared and thermal testing: technique of infrared thermography," Nondestructive Testing Handbook Series 3rd Ed, X. P. V. Maldague, P. O. Moore Ed, ASNT, Columbus, USA, PP. 318-328. 2001

[2] M. Y. Choi, K. S. Kang, J. H. Park, W. T. Kim and K. S. Kim, "Defect sizing and location by lock-in photoinfrared thermography," Journal of the Korean Society for Nondestructive Testing, Vol. 27, No. 4, PP. 321-327. 2007

[3] H. S. Park, M. Y. Choi, J. H. Park, W. T. Kim, W. J. Choi, "Study on the Qualitative Defects Detection in Composites by Optical Infrared Thermography", Journal of the Korean Society for Nondestructive Testing, Vol. 31, No. 2. 2011 